\title{
SIMPLE SUBRINGS OF ALGEBRAS OVER FIELDS
}

\author{
JAN KREMPA
}

\begin{abstract}
In this note we shall prove that if $A$ is a not necessarily associative algebra over a field $K$ and $R$ is a simple subring of $A$ with centroid $F$ then $\operatorname{dim}_{F} R<\operatorname{dim}_{K} A$.

Since we do not use polynomial identities in a proof of this result then we have obtained an affirmative answer to the 11 th question from [2], posed by $\mathbf{I}$. $\mathrm{N}$. Herstein.
\end{abstract}

In this paper $A$ denotes an algebra over a field $K, R$ is a simple (idempotent) subring of $A$ and $F$ denotes a centroid of $R$. It is well known [1] that $F$ is a field and $R$ is an $F$-algebra.

Theorem. $\operatorname{dim} F_{R}<\operatorname{dim}_{K} A$.

Proof. Let $A_{1}=K R$ be the $K$-subspace of $A$ spanned by $R$. It is obvious that $A_{1}$ is a $K$-subalgebra of $A$ containing $R$. Now let $M$ be a maximal element of a family of ideals $J$ of a ring $A_{1}$ such that $J \cap R=0$. Put $B=A / M$. Since $R$ is a simple ring then it is easy to see that $M$ is a prime ideal of $A_{1}$ and consequently it is an ideal of the $K$-algebra $A_{1}$. Thus $B$ is a $K$-algebra containing $R$ in a natural way. Now if $I$ is a nonzero ideal of an algebra $B$ then by the construction of $B$ it follows that $I \cap R=R$. Now $R^{2}=R$ and $B=K R$ implies that $I=B$, i.e. $B$ is a simple algebra. Let $L$ denote a centroid of $B$. Obviously we get the following inequalities

$$
\operatorname{dim}_{K} A>\operatorname{dim}_{K} A_{1}>\operatorname{dim}_{K} B>\operatorname{dim}_{L} B .
$$

Let $f \in F, l_{i} \in L, r_{i} \in R$ and $s \in R$. Assume that $\Sigma l_{i} r_{i}=0$. Then we have $\left(\sum l_{i}\left(f r_{i}\right)\right) s=\sum l_{i}\left(\left(f r_{i}\right) s\right)=\sum l_{i}\left(r_{i}(f s)\right)=\left(\sum l_{i} r_{i}\right)(f s)=0$. Then $\left(\sum l_{i}\left(f r_{i}\right)\right) R=0$. Since $R$ generates $B$ as a $K$-linear space then $\left(\sum l_{i}\left(f r_{i}\right)\right) B=0$. Analogously from the equality $s\left(\sum l_{i}\left(f r_{i}\right)\right)=(f s)\left(\sum l_{i} r_{i}\right)=0$ it follows that $B\left(\sum l_{i}\left(f r_{i}\right)\right)=0$. Since $B$ is a simple ring then we see that $\Sigma l_{i}\left(f r_{i}\right)=0$. Hence it follows that the rule $g(f) \cdot \sum l_{i} r_{i}=\sum l_{i}\left(f r_{i}\right)$ defines unambigously the extension of action $f$ on $R$ to the action $g(f)$ on $B$. It is easily seen that $g$ is an imbedding of $F$ into $L$. In this way $L$ and $B$ become algebras over $F$.

Now we are able to define a natural homomorphism $h: L \otimes_{F} R \rightarrow B$ defined by the rule $h\left(\sum l_{i} \otimes r_{i}\right)=\sum l_{i} r_{i}$. Since $h$ is a nonzero homomorphism and $L \otimes_{F} R$ is a simple ring (see [1, p. 292]) then $h$ is an injection of $K:=L$ algebras. Thus we have obtained

$$
\operatorname{dim}_{F} R=\operatorname{dim}_{L} L \otimes_{F} R<\operatorname{dim}_{L} B .
$$

Received by the editors March 26, 1980.

1980 Mathematics Subject Classification. Primary 16A56.

(C) 1981 American Mathematical Society 0002-9939/81/0000-0151/\$01.50 
This together with (1) ends the proof of the theorem.

Observe that by the proof of the theorem we get the following

Corollary. If $\operatorname{dim}_{K} A=\operatorname{dim}_{F} R<\infty$ then $A$ is a central simple $K$-algebra isomorphic to $K \otimes_{F} R$.

\section{REFERENCES}

1. N. Jacobson, Lie algebras, Interscience, New York, 1962.

2. F. Van Oystaeyen (Ed.), Ring theory (Proc. 1978 Antwerp Conf.), Marcel Dekker, New York, 1979.

mathematics Institute, University of Warsaw, Warsaw, Poland 